\title{
SUR UN CYSTICERCUS FASCIOLARIS RUDOLPHI TÉRATOLOGIQUE (POLYCÉPHALE) (1)
}

\author{
Par Robert-Ph. DOLLFUS
}

A la dissection d'une souris blanche $\delta^{*}$, morte au laboratoire de Yaoundé (Cameroun), le médecin-capitaine J. Guibert trouva (11-11937), dans l'hypocondre gauche, une masse globuleuse, de la grosseur d'une noisette, adhérente à la paroi et aux organes voisins, foie, rate et surtout intestin. A l'intérieur de cette formation pathologique, il y avait, baignant dans un liquide clair, " eau de roche », un cystique polycéphale vivant, dont les scolex étaient évaginés. Les scolex s'étiraient et se contractaient; à chaque contraction, la vésicule centrale se gonflait par apport du liquide contenu dans la partie non segmentée de chaque branche.

Conservé en eau physiologique formolée, ce cystique a été envoyé, par le $\mathrm{D}^{\mathrm{r}} \mathrm{J}$. Guibert, au Professeur Emile Brumpt, qui me l'a aimablement communiqué pour identification.

Description morphologique. - La vésicule (fig. 1), gonflée de liquide, a assez exactement la forme d'un haricot (longueur $13 \mathrm{~mm}$., largeur au niveau du hile $7 \mathrm{~mm}$., 5, épaisseur $7 \mathrm{~mm}$., environ), elle est molle et déformable par pression légère ; de sa périphérie, naissent perpendiculairement, dans un même plan, six pédoncules terminés chacun par une double couronne de crochets, entourée de quatre ventouses. Les branches sont longues de 10-12 mm., avec une largeur d'environ $3 \mathrm{~mm}$., sauf au point d'attache, rétréci jusqu'à 1,5-2 mm. ; elles ne sont pas tout à fait cylindriformes, il y a un léger aplatissement et l'union des faces est indiquée par deux légères saillies longitudinales opposées, visibles sur les coupes transversales.

La partie distale de chaque branche est solide (sur environ 4-5 mm.). avec un faible plissement transversal (segmentation) assez régulier; sa coloration est jaunâtre, alors que la partie proximale, remplie par le liquide vésiculaire, est, comme la vési-

(1) Récolté par le $\mathrm{D}^{\mathrm{r}} \mathrm{J}$. Guibert au Cameroun.

Annales de Parasitologie, T. XVI, No $2,-1^{\text {er }}$ mars 1938, p. $133-141$. 
cule centrale, translucide: d'un blanc-bleuâtre sur fond noir, blanche sur fond blanc.

L'extrémité d'une branche a été transversalement sectionnée un peu au-dessous du niveau des ventouses. La figure 2 montre l'aspect, en projection sur un plan, de cette extrémité, vue par l'apex, avec sa double couronne de 23 grands crochets et 23 petits. Dans cette position, l'axe longitudinal des crochets étant oblique par rapport au plan, il n'est pas possible de se rendre compte exactement de la forme et des dimensions de ceux-ci ; par un effet de perspective, les grands crochets présentent un contour en forme de biscuit, les

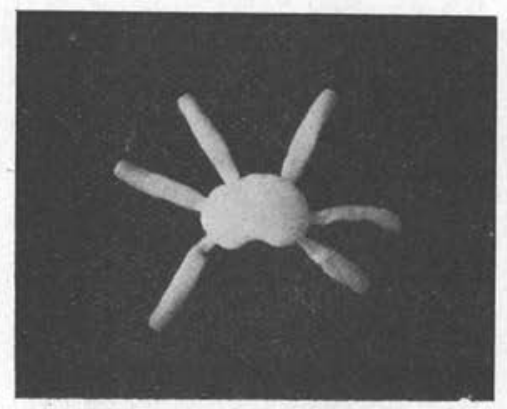

Fig. 1. - Cystique de J. Guibert. Photographie un peu réduite.

petits crochets semblent avoir une extrémité très élargie, presque spatulée, de plus, la garde n'est reconnaissable ni pour les grands crochets, ni pour les petits. Dégagés de leur musculature et isolés par dissociation au moyen de fines aiguilles, sous le microscope, les crochets ont été observés sous leur forme exacte et mesurés.

Les grands crochets (fig. 3) ont une longueur totale de 395 à $399 \mu$, la lame est nettement plus courte que le manche, la garde forme une protubérance très marquée, ovale vue de face, et sans bilobation.

Les petits erochets ont une longueur totale de 244 à $255 \mu$, la lame est nettement plus longue que le manche, la garde forme une protubérance très marquée, arrondie vue de face et sans bilobation.

Les ventouses, rétractées et un peu déformées, mesurent environ $300 \mu \times 200 \mu$.

Identification. - D'après les caractères du scolex, il est évident qu'il s'agit d'une espèce de la famille des Tæniadæ; il est naturel de chercher d'abord des termes de comparaison parmi les espèces à cystique normalement polycéphale (Cœnurus-Multiceps), une dizaine 
environ sont connues; il n'y en a pas dont les grands crochets dépassent $180 \mu$ et les petits $150 \mu$, il n'y en a pas dont le cystique, par son habitus, rappelle celui trouvé par le $\mathrm{D}^{\mathrm{r}} \mathrm{J}$. Guibert.

Nous devons donc chercher parmi les Tænia à cystique normalement monocéphale ou à cystique inconnu ; une vingtaine d'espèces

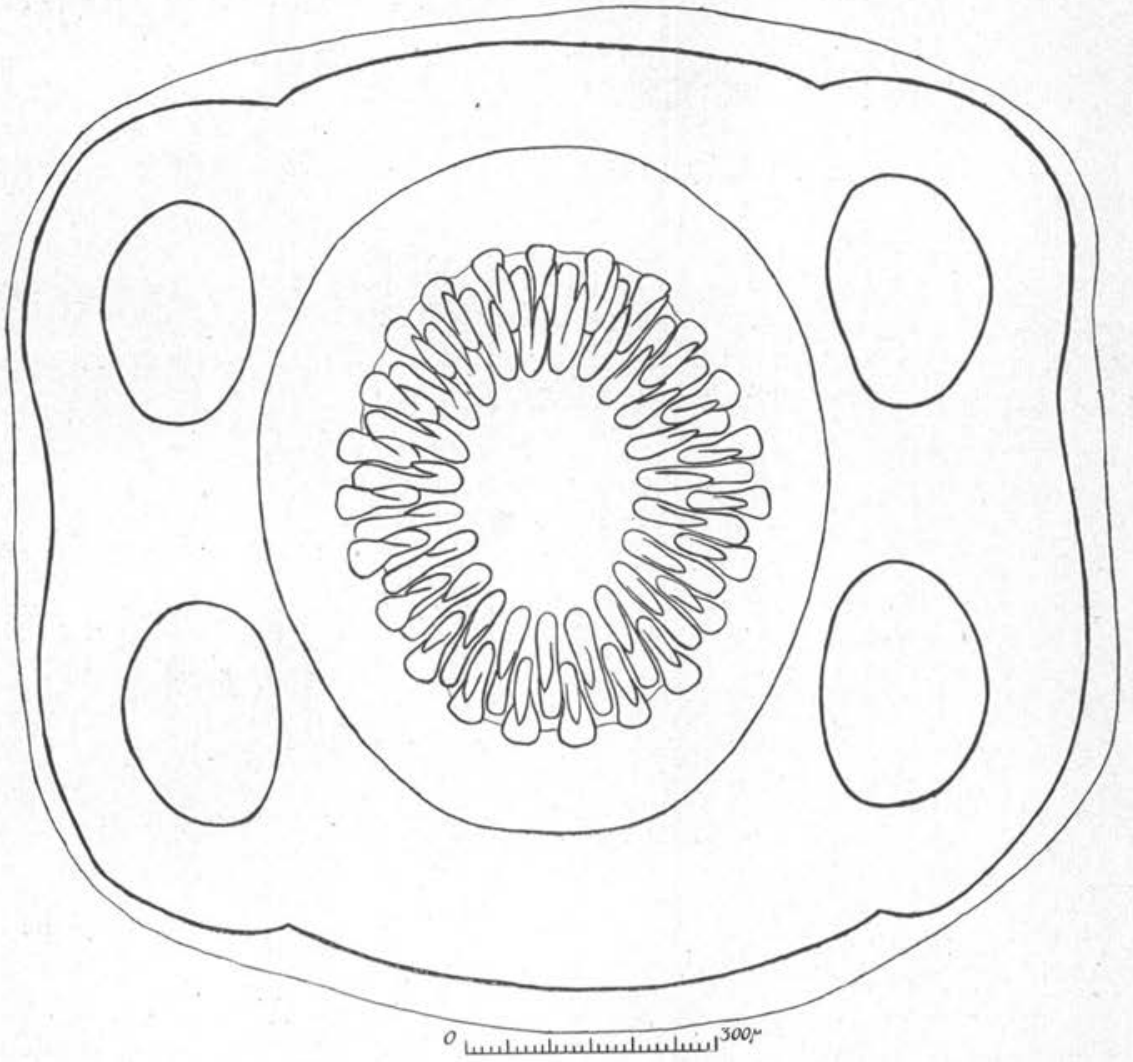

FIG. 2. - Cystique de J. Guibert, apex d'une des branches, préparation in toto, les crochets sont obliques par rapport au plan du dessin, ils n'apparaissent pas sous leur forme exacte, leur garde n'est pas discernable.

environ sont décrites, parmi lesquelles deux seulement, d'après le nombre, la forme et les dimensions des crochets, peuvent être prises en considération : tæniæformis Batsch 1786 et laticollis Rud. 1819. Nous éliminons naturellement Tænia infantis J. Bacigalupo 1922 (indiqué comme expulsé par un enfant de 5 ans à Buenos-Ayres et non retrouvé chez l'homme); Bacigalupo lui-même, puis Ch.-W. Stiles et M. Orleman (1925, p. 250), ont noté la grande ressemblance 
de $T$. infantis Bacig. avec $T$. tæniæformis Batsch. De plus, aucun caractère d'infantis ne permettant de le séparer de tæniæformis, l'on admet actuellement, avec Ch. Joyeux et J.-G. Baer (1929, p. 133) (cf. E. Brumpt, 1936, p. 779), qu'infantis est seulement un synonyme à ajouter à la liste, déjà longue, de ceux de tæniæformis-crassicollis. Pour T. laticollts Rud., adulte chez les Lynx et Genetta en Europe,
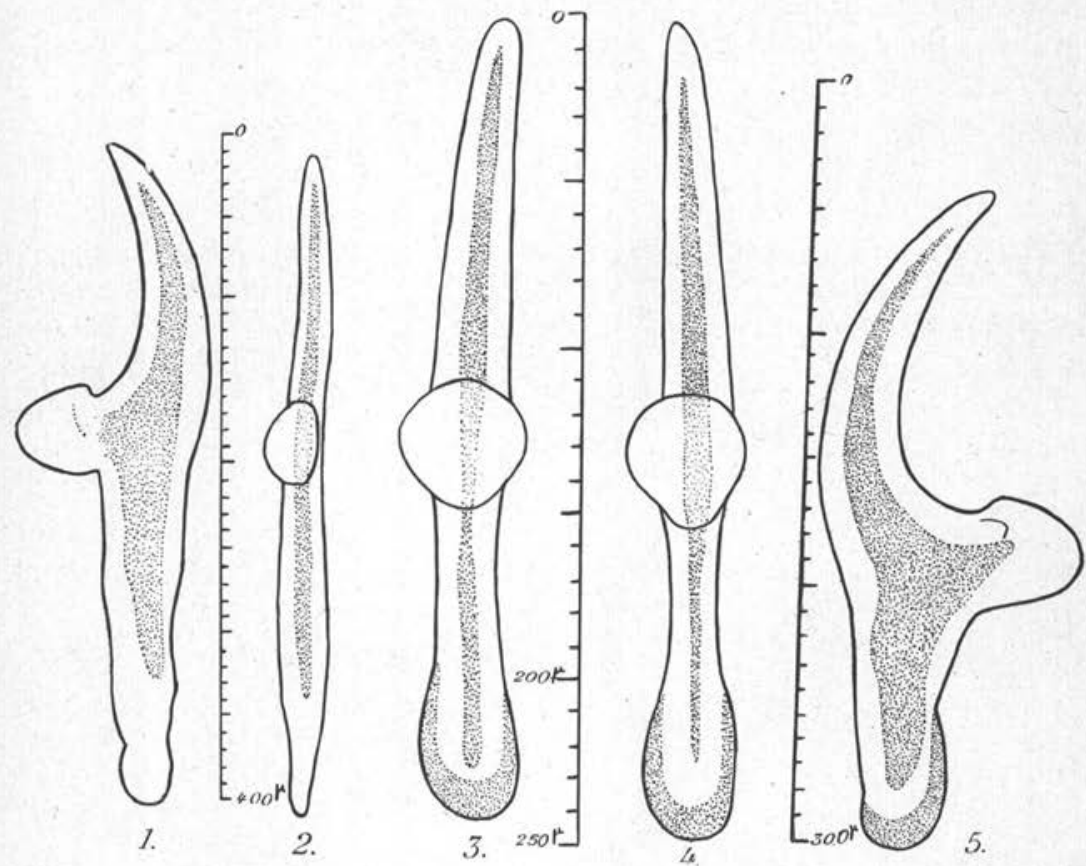

FIG. 3. - 1 et 2 , deux grands crochets isolés du même cystique ; 3, 4, 5, petits crochets isolés du même cystique.

chez les Lynx en Amérique du Nord, les ouvrages classiques mentionnent 38 à 60 crochets, dont les grands sont longs de 380 à $420 \mu$, les petits de 150 à $183 \mu$. Mais, chez les spécimens de cette espèce récemment étudiés par Mary Scott Skinker (1935, p. 216219 , pl. XIX, fig. 1, crochet d'après Leuckart, fig. 8, crochets d'après Skinker), il y a 38 à 42 crochets, dont les grands sont longs de 390 à $415 \mu$ et les petits de 214 à $238 \mu$; la garde est, légèrement seulement, bilobée.

Les grands crochets de laticollis ont, à très peu près, la forme de ceux du cystique trouvé par le $\mathrm{D}^{\mathrm{r}} \mathrm{J}$. Guibert, mais les petits crochets en diffèrent nettement ; ils sont, dans l'ensemble, plus 
petits, leur manche n'a pas la même forme, il est beaucoup plus court; l'axe du manche fait, avec l'axe de la garde, un angle très obtus, alors que cet angle est presque un angle droit pour les petits crochets du cystique trouvé par le $\mathrm{D}^{\mathrm{r}} \mathrm{J}$. Guibert.

Pour $T$. tæniæformis Batsch, adulte chez de nombreuses espèces de Félidés et Mustélidés en Europe, Asie, Amérique du Nord et Amérique du Sud, on trouve mention de 26 à 52 crochets, dont les grands sont longs de 380 à $420 \mu$, et même $430 \mu$, les petits de 250 à $270 \mu(1)$; généralement, il $\mathrm{y}$ a de 34 à 48 crochets dont la longueur s'éloigne peu (en plus ou en moins) de $400 \mu$ pour les grands et de 245-255 $\mu$ pour les petits ; la garde des grands crochets, d'après Maurice-C. Hall (1919, p. 10), présente « a tendency to bifid structure ». De très nombreuses figures des crochets de tæniæformis ont été publiées, elles ne sont pas toutes, évidemment, superposables et il y a de petites variations (2), portant principalement sur la grosseur du manche, la terminaison conique ou arrondie de la garde, la bilobation de la garde. Au sujet de la bilobation de la garde, il est utile de remarquer que, chez les diverses espèces de Tænia où elle a été observée, elle est due à une encoche longitudinale plus ou moins étendue : celle-ci fait paraître la garde, vue de face, cordiforme, lorsqu'elle affecte seulement le bord supérieur, ou en arceau, lorsqu'elle affecte seulement le bord inférieur.

(1) Max Lühe $(1910$, p. 704$)$ admet 36 crochets de $400 \mu$ et $250 \mu$ et il donne un tableau minutieux de toutes les dimensions de toutes les parties des crochets (14-15 mesures par crochet !) de taniaformis-crassicollis. Aux EtatsUnis, A.-E. Loveland $(1894$, p. 80 ) a compté $34-36$ crochets de $320-420 \mu$ et 180 $280 \mu$, il n'a pas donné de renseignements sur leur forme.

Il n'est pas croyable que le nombre des crochets soit plus élevé chez l'adulte que chez le cystique, cependant, dans sa monographie de C. fasciolaris Rud., Ernst Bartels (1902, p. 514) n'a pas hésité à écrire : « Le nombre des crochets par couronne est de 17 à 18 chez C. fasciolaris, alors qu'il est de 20 à 24 chez Tania crassicollis, fait qui a déjà été établi par Küchenmeister, 1852 »!

Küchenmeister (1852, p. 138-139-141) dit, en effet, qu'il a compté, chez C. fasciolaris de la souris, 18 crochets de $403 \mu$ et 18 de $245 \mu$, chez $T$. crassicollis du chat 24 à 26 crochets de $426 \mu$ et 24 à 26 de $268 \mu$; il en a déduit que le nombre des crochets augmentait avec l'âge du parasite, ce qui n'a jamais été confirmé. La variation numérique observée par Küchenmeister est comprise dans les limites de l'espèce et e'est, apparemment, par suite d'un hasard que les fasciolaris de Küchenmeister se sont trouvés avoir moins de crochets que les adultes.

(2) Comparer, par exemple, entre elles les figures données par Küchenmeister (1852, fig. XV grand crochet, XVI petit crochet de C. fasciolaris de la souris ; fig. XVII $a-b$ grand crochet et XVIII $a-b$, petit crochet) de $T$. crassicollis du chat.

Une bonne description des crochets de crassicollis du chat a aussi été donnée par Rudolf Leuckart (1856, p. 55-56, pl. II, fig. $1 a$ : un des 22-26 grands crochets longs de $0 \mathrm{~mm}$. 39 ; fig. $1 b$ : un des $22-26$ petits crochets), mais sans préciser la longueur totale des petits crochets; si lon calcule cette longueur totale : d'après le grossissement indiqué, on obtient 0,229 ; d'après le nombre indiqué pour la distance entre l'extrémité de la base et l'extrémité de la garde, on obtient 0,292 ; par comparaison avec la figure représentant à lạ même échelle le grand crochet, on obtient 0,240 : 
Il ne semble pas que cette êncoche soit toujours constante et bien marquée chez toutes les espèces. A propos de $T$. omissa Lühe, Max Lühe (1910, p. 696) dit que la profondeur de cette encoche, qui existe aussi chez maints autres Cystotænia, peut être très variable, non seulement chez les différents individus, mais aussi dans une même couronne et que l'on peut même observer seulement une très légère délimitation concave au lieu d'une entaille nette.

J'ai personnellement examiné quelques couronnes de crochets de
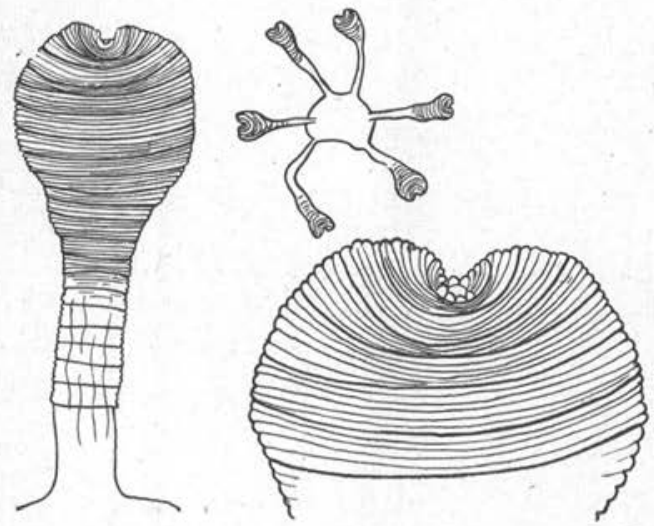

FiG. 4. - Croquis originaux du $\mathrm{D}^{\mathrm{r}} \mathrm{J}$. Guibert montrant l'habitus du eystique in vivo et l'aspect plissé d'une des branches.

T. tæniæformis Batsch, à titre de comparaison. Chez un adulte obtenu expérimentalement par E. Brumpt (36 crochets; grands $400 \mu$; petits $245-248 \mu$ ), je n'ai pas réussi à voir de crochets à garde nettement bilobée; la garde de quelques grands erochets montrait tout au plus un léger indice de bilobation.

Ce caractère de l'encoche de la garde mis à part, les crochets du cystique du $\mathrm{D}^{\mathrm{r}} \mathrm{J}$. Guibert correspondent mieux à ceux de T. tæniæformis Batsch qu'à ceux de n'importe quelle autre espèce de Tæniadæ; de plus, le fait que chaque scolex est porté par un long pédoncule subcylindrique segmenté concorde avec ce qui existe chez Cysticercus fasciolaris Rud. ; donc, à moins de supposer que ce cystique est celui d'une espèce encore inconnue ou insuffisamment décrite, je suis amené à conclure qu'il s'agit bien de tæniæformis et que la polycéphalie est tératologique (1). Quel

(1) Si j'avais eu à examiner une seule des branches segmentées, avec fragment de vésicule, du cystique de J. Guibert, sans avoir été informé que ce cystique était polycéphale, je n'aurais eu aucune hésitation à l'identifier immédiate- 
facteur a provoqué la polycéphalie, celle-ci est-elle héréditaire ? de cela, nous ne savons absolument rien.

Si exceptionnel que soit ce cystique tératologique, il n'est pas le seul connu qui se présente sous cette forme (1). Nous devons à T. Southwell et A. Kirshner (1937, p. 37-42, fig. 1-3) l'étude d'un cystique de même habitus, morphologiquement très voisin, mais à douze branches, trouvé à Sierra-Léone, chez Mastomys erythroleucus (Temminck 1855), attaché par un pédicule à la surface péri-
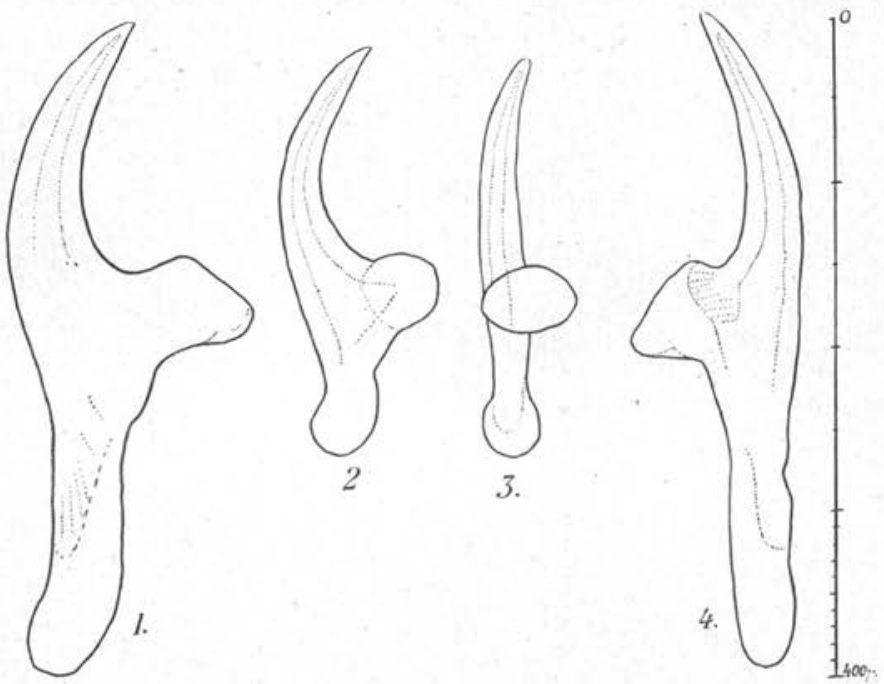

FiG. 5. - Grands et petits crochets d'un Twnia taniaformis Batsch, obtenu expérimentalement chez une chatte par le Professeur E. Brumpt (15-5-1908).

tonéale de la rate. Les douze branches segmentées s'étalent dans un même plan, elles sont longues de 20-22 mm., larges de $2 \mathrm{~mm}$., 2 à $2 \mathrm{~mm}$., 5, et rayonnent d'une vésicule transparente, globuleuse, de $10 \mathrm{~mm}$. de diamètre. Chaque scolex porte, en avant de ses quatre ventouses, une double couronne de crochets : 22 grands, longs de 380 à $400 \mu$, et 22 petits, longs de 220 à $240 \mu$.

ment à $C$. fasciolaris Rud. (genre Hydatigera Lamarck $1816=$ Reditænia Sambon 1924). Les crochets et ia morphologie externe sont suffisamment caractéristiques pour qu'il soit superflu de chercher une confirmation dans l'anatomie fine (musculature, système nerveux, appareil excréteur), en comparant celle d'une des branches du cystique de Guibert à celle, bien connue, d'un C. fasciolaris Rud. monocéphale.

(1) Au Laboratoire de Parasitologie de la Faculté de Médecine de Paris, où l’on a disséqué et examiné, pour leurs parasites, des milliers de souris et de rats, aucun cystique de cette forme n'a été trouvé. 
T. Southwell et A. Kirshner ont comparé avec soin ce cystique aux cœnures et autres cystiques polycéphales connus, puis aux divers Trnia à grands crochets, ils sont arrivés à cette conclusion que chacune des branches segmen'ées est indistinguible de Cysticercus fasciolaris Rud.

Entre les crochets du cystique de Mastomys et ceux figurés par M.-C. Hall (1919) pour $T$. tæniæformis Batsch, il y a évidemment de petites différences que Southwell et Kirshner ont considérées comme une variation normale dans les limites de l'espèce. Entre les crochets du cystique de Mastomys et ceux du cystique de souris de J. Guibert, il y a aussi de petites différences, mais qui paraissent bien être aussi comprises dans les limites de la variation de l'espèce; la différence la plus marquée est dans la forme de la garde ; elle est, vue de face, à contour triangulaire, avec concavité du bord antérieur, « distinctly bifid when viewed from the anteroposterior aspect », disent Southwell et Kirshner, en accord avec leur figure $2 c$.

J'ai exposé plus haut que, chez fasciolaris-tæniæformis, le caractère bilobé de la garde ne semblait être ni constant, ni caractéristique; j'estime donc que ce caractère ne peut pas être invoqué pour séparer spécifiquement le cystique polycéphale de J. Guibert, ni de celui de Southwell et Kirshner, ni de C. fasciolaris Rud.

\section{RÉSUMÉ}

Un cystique polycéphale a été trouvé chez une souris blanche de laboratoire à Yaoundé (Cameroun) par le Médecin-Capitaine J. Guibert. Ce cystique à six branches segmentées, rayonnant dans un même plan à partir de la vésicule, est du même type qu'un cystique à douze branches trouvé chez un Mastomys à Sierra-Leone et étudié par T. Southwell et A. Kirshner (1937).

Dans l'un et l'autre cas, chacune des branches, considérée isolément, présente les mêmes caractéristiques morphologiques (segmentation; nombre, forme et dimensions des crochets) que Cysticercus fasciolaris Rud., larve de Tænia tæniæformis Batsch $=T$. crassicollis Rud.

Nous concluons que le cystique de ce Tænia, normalement monocéphale, peut, tératologiquement, se présenter sous une forme polycéphale. 


\section{BIBLIOGRAPHIE}

* Bacigalupo (J.). - Tania infantis n. sp. Semana medica, Buenos-Aires, XIX, 1922, p. $302-305$, fig. $1-5$.

Bantels (E.). - Cysticercus fasciolaris. Anatomie. Beiträge zur Entwicklung und Umwandlung in Trnia crassicollis. Zoolog. Jahrbücher. Anat., XVI, 1902, p. 511-579, fig. texte A-B, pl. XXXVII-XXXIX, fig. 1-30.

Brumpt (E.). - Précis de Parasitologie. 5e édition. Paris, Masson et Cie, 1936.

HALr. (M. C.). - The adult tænioid cestodes of dogs and cats, and of related carnivores in North America. Proceed. Un. St. Nat. Mus., LV, ${ }^{\circ}$ 2258, p. 1-94, fig. 1-80.

Joyeux (Ch.) et Baer (J. G.). - Les cestodes rares de l'homme. Bull. Soc. Pathologie exotique, XXII, 1929, p. 114-136.

Küchenmeister (F.). - Ueber die Umwandlung der Finnen (Cysticerci) in Bandwürmer (Tænien). Vierteljahrsschrift für die prakt. Heilkunde. Prag., IX Jahrg (1852), I Bd. (XXXIII Bd), p. 106-158, pl. fig. I-XXVI.

Levckart (R.). - Die Blasenwürmer und ihre Entwicklung. Giessen 1856,

Loveland (A. E.), - On the anatomy of Tania crassicollis Rud. Journ. Comparat. Med. and Veterin. Archives. Philadelphia, XV, 1894, p. 67-89, pl. IIV, fig. 1-18. Supplementary Note by C. W. Stiles, p. 85.

LüHE (M.). - Cystotänien südamerikanischer Feliden. Zoolog. Jahrbücher, Supplement 12, Heft 3, 1910, p. 687-710, fig. texte A-H, pl. XIX-XX, fig. 1-12.

Skinker (M. S.). - Two new species of Tapeworms from Carnivores and a redescription of Trenia laticollis Rudolphi, 1819. Proceed. Un. St. Nat. Mus., LXXXIII, $\mathrm{n}^{\circ} 2980$, 1935, p. 211-220, pl. XIX-XXI.

Southwell (T.) et Kirshner (A.). - Description of a polycephalic Cestode Larva from Mastomys erythroleucus, and its probable identity. Annals of Trop. Medic. and Parasitol., Liverpool, XXXI, 1937, p. 37-42, fig. 1-3.

Stiles (Ch. W.) et Orleman (M.). - The Cestode genus Hydatigera Lamarck 1816, species reditania Sambon, 1924. Journ. of Tropical Medicine and Hygiene, XXVIII, 1925, p. 249-250.

Institut de Parasitologie de la Faculté de Médecine de Paris (Directeur : Prof. Emile Brumpt) et Laboratoire des Productions coloniales du Muséum de Paris (Directeur : Prof. Abel Gruvel). 